\title{
Survey of modeling, analysis, and control of hybrid systems*
}

\author{
B. De Schutter, W.P.M.H. Heemels, J. Lunze, and C. Prieur
}

If you want to cite this report, please use the following reference instead:

B. De Schutter, W.P.M.H. Heemels, J. Lunze, and C. Prieur, "Survey of modeling, analysis, and control of hybrid systems," Chapter 2 in Handbook of Hybrid Systems Control - Theory, Tools, Applications (J. Lunze and F. Lamnabhi-Lagarrigue, eds.), Cambridge, UK: Cambridge University Press, ISBN 978-0-521-76505-3, pp. 31-55, 2009.

Delft Center for Systems and Control

Delft University of Technology

Mekelweg 2, 2628 CD Delft

The Netherlands

phone: +31-15-278.24.73 (secretary)

URL: https: / / www.dcsc.tudelft.nl

*This report can also be downloaded viahttps://pub. deschutter.info/abs/09_007.html 


\title{
Survey of modeling, analysis, and control of hybrid systems
}

\author{
B. De Schutter ${ }^{1}$, W. P. M. H. Heemels ${ }^{2}$, J. Lunze ${ }^{3}$, and C. Prieur ${ }^{4}$
}

1 Delft Center for Systems and Control \& Marine and Transport Technology, Delft University of Technology, Mekelweg 2, 2628 CD Delft, The Netherlands, b.deschutter@dcsc.tudelft.nl

2 Eindhoven University of Technology, Mechanical Engineering, Control Systems Technology, PO Box 513, 5600 MB Eindhoven, The Netherlands, W.P.M.H.Heemels@tue.nl

3 Institute of Automation and Computer Control, Department of Electrical Engineering and Information Sciences, Universitaetsstrasse 150, D-44780 Bochum, Germany, lunze@atp.rub.de

4 LAAS-CNRS, Université de Toulouse, 7, avenue du Colonel Roche, 31077

Toulouse, France, Christophe.Prieur@laas.fr

An overview of various modeling frameworks for hybrid systems is given followed by a comparison of the modeling power and the model complexity, which can serve as a guideline for choosing the right model for a given analysis or control problem with hybrid dynamics. Then, the main analysis and design tasks for hybrid systems are surveyed together with the methods for their solution, which will be discussed in more detail in subsequent chapters.

\subsection{Models for hybrid systems}

\subsubsection{Overview}

As models are the ultimate tools for obtaining and dealing with knowledge, not only in engineering, but also in philosophy, biology, sociology, and economics, a search has been undertaken for appropriate mathematical models for hybrid systems. This section gives an overview of the modeling formalisms that have been elaborated in hybrid systems theory in the past.

Structure of hybrid systems. Many different models have been proposed in literature as will be seen in the next chapters. These models can be distinguished with respect to the phenomena that they are able to represent in an 
explicit form. Consequently, these models have different fields of applications. The main idea of these models is described by the block diagram shown in Fig. 1.1, which is often used in literature as a starting point of hybrid systems modeling and analysis although not all models use this structure in a direct way.

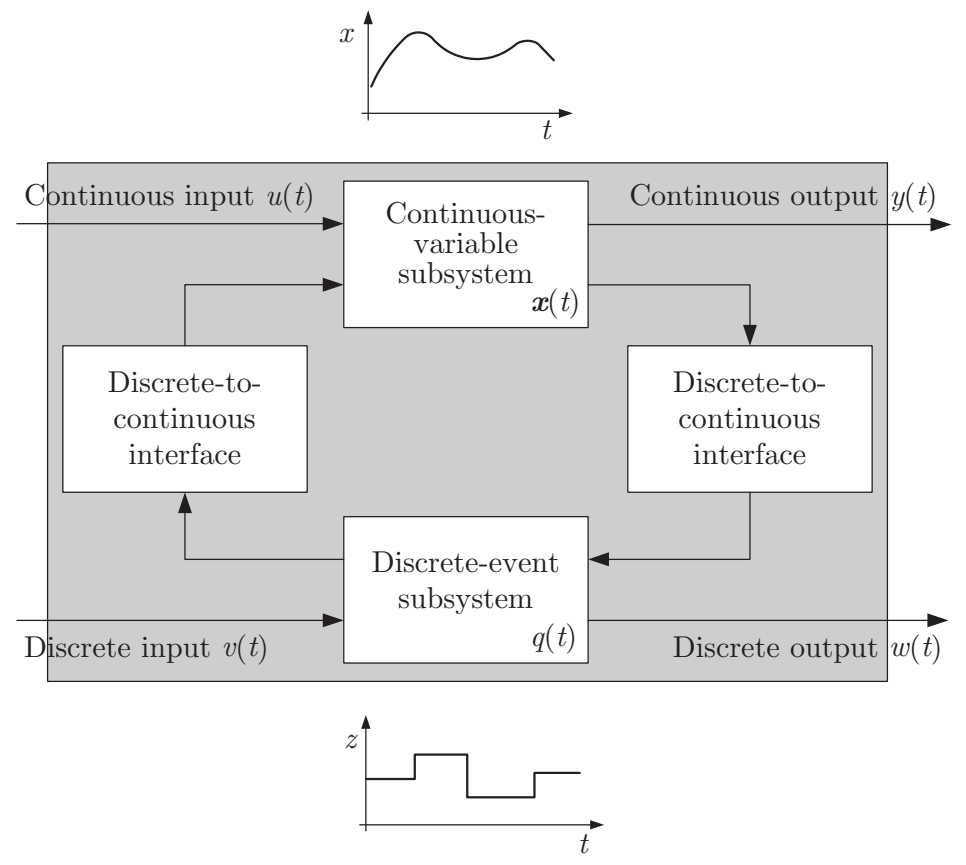

Fig. 1.1. Structure of hybrid systems

The hybrid character of the system is reflected in this structure by the combination of a continuous-variable system shown in the upper part of the figure and a discrete-event system below. The continuous input and the continuous output are associated with the continuous subsystem and the discrete signals with the discrete subsystem. The continuous subsystem describes how the continuous state $\boldsymbol{x}(t)$ of the hybrid system develops over time. The discrete subsystem characterizes the evolution of the discrete state $q(t)$.

As the signals occurring in both subsystems have different signal spaces, an interconnection between both model parts necessitates two interfaces, which are called the discrete-to-continuous interface or the continuous-to-discrete interface, respectively. The discrete-to-continuous interface associates with a discrete signal value that is generated by the discrete-event subsystem a continuous-valued signal that acts as an input of the continuous subsystem. This interface will is also called injector. The continuous-to-discrete interface 
transforms a continuous-variable signal into a signal with discrete signal space. It typically tests whether the continuous signal has exceeded a given threshold or, more generally, whether the continuous state $\boldsymbol{x}(t)$ has reached a switching surface. The result is an event, which is described by the event name and the event time instant. Therefore, this interface is often called an event generator and are formed by guards and invariants in hybrid automata.

The models described below distinguish with respect to the way in which they represent the two kinds of subsystems shown in the figure and the interfaces.

Models for hybrid systems. A whole range of possible model structures for hybrid systems has already been proposed of which we present a partial list below:

- hybrid automata,

- switched systems,

- piecewise linear or piecewise affine models,

- timed automata and timed or hybrid Petri nets,

- differential automata,

- mixed logical dynamic models,

- real-time temporal logics and timed communicating sequential processes,

- complementarity systems,

- hybrid inclusions.

We would like to emphasize that this list is by no means exhaustive and that we will not discuss all these classes here, but we will focus on the most wellknown classes, which will also be treated in more detail in the next chapters of this handbook. Additional references to other hybrid modeling formalisms not discussed here will be provided in the bibliographical notes.

The common feature of all the modeling paradigms and in fact of hybrid systems in general is the interaction of different dynamics. This indicates that also the model structure should mix two modeling formalisms. Typically, one might think of the interaction of time-driven models (governed by differential or difference equations) on one hand, and event-driven systems (described by, e.g., temporal logic, automata, finite-state machines, etc.) or logic rules on the other hand. In some way these features should be combined in one model structure. One generally accepted manner of looking at hybrid systems is via hybrid automata, which can be seen as a cross product of finite-state machines and differential or difference equations (depending one whether a discrete-time or continuous-time formalism is used).

\subsubsection{Hybrid automata}

Hybrid automata result as an extension of finite-state machines by associating with each discrete state a system of differential or difference equations that describe how in this discrete state the continuous state evolves. 
We would like to stress that it can be a nontrivial task to rewrite a physical model description in terms of a hybrid automaton. Especially, the definition of the guards, the invariant sets, and the reset maps (i.e., switching and reinitialization rules) can be really involved.

\subsubsection{Switched systems}

A quite general class of hybrid systems concerns switched systems given by

$$
\dot{\boldsymbol{x}}(t)=\boldsymbol{f}_{q(t)}(\boldsymbol{x}(t)),
$$

where $\boldsymbol{x} \in \mathbb{R}^{n}$ denotes the state and $q: \mathbb{R}_{+} \rightarrow\{1, \ldots, N\}$ is the switching signal that determines which vector field $\boldsymbol{f}_{q}$ with $q \in\{1, \ldots, N\}$ is active at time $t \in \mathbb{R}_{+}$.

For a fixed $q,(1.1)$ describes a non-switched system, which is sometimes called the subsystem of a switched system. In this context, switching means the currently active subsystem is changed to another one.

The switching can depend on time only as above, but it can also be a function of the state $\boldsymbol{x}(t)$ at time $t$ or of an external input, and it can even have memory in it.

In particular, when the switching only depends on the state variable $\boldsymbol{x}(t)$ at the present time $t$, one speaks of discontinuous dynamical systems or piecewise smooth systems. An example is the system below, which switches between two dynamics as a result of inequalities in the state variable:

$$
\dot{\boldsymbol{x}}(t)=\boldsymbol{f}(\boldsymbol{x}(t))= \begin{cases}\boldsymbol{f}_{-}(\boldsymbol{x}(t)), & \text { if } \phi(\boldsymbol{x}(t))<0 \\ \boldsymbol{f}_{+}(\boldsymbol{x}(t)), & \text { if } \phi(\boldsymbol{x}(t))>0 .\end{cases}
$$

\section{$\boldsymbol{C}_{+}$}

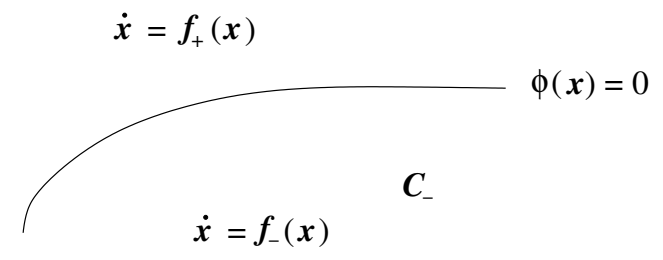

Fig. 1.2. Switching dynamics.

The state space is separated into two parts by a hyper-surface defined by $\phi(\boldsymbol{x})=0$ (see Fig. 1.2). On one side of the surface $\mathcal{C}_{+}:=\left\{\boldsymbol{x} \in \mathbb{R}^{n} \mid \phi(\boldsymbol{x})>0\right\}$ the dynamics $\dot{\boldsymbol{x}}=\boldsymbol{f}_{+}(\boldsymbol{x})$ holds, on the opposite side $\mathcal{C}_{-}:=\left\{\boldsymbol{x} \in \mathbb{R}^{n} \mid \phi(\boldsymbol{x})<\right.$ $0\}$ the dynamics $\dot{\boldsymbol{x}}=\boldsymbol{f}_{-}(\boldsymbol{x})$ is valid. Hence, one can also consider this system as a differential equation with a discontinuous right-hand side [Filippov, 1988]. 
As the choice of whether the vector field $\boldsymbol{f}_{-}$or $\boldsymbol{f}_{+}$is active at time $t$ only depends on the state $\boldsymbol{x}(t)$, no discrete state is necessary to describe such systems. In the light of the description of hybrid systems given in Chapter 1 of this handbook systems of this class are no "real" hybrid system. However, they cannot be analyzed by standard methods elaborated for nonlinear systems, because the Lipschitz continuity of the vector field $\boldsymbol{f}$ is lost at the switching surface and, consequently, the definition of solutions and well-posedness have to be extended with respect to nonlinear systems. Therefore, new methods for dealing with such systems have been developed in the field of hybrid dynamical systems, and $q$ is said to be the discrete state or the operation mode of the system.

\subsubsection{Piecewise affine systems}

If the discontinuous dynamical system has affine dynamics in each region and the regions are polytopic, we obtain the well-studied class of piecewise affine (PWA) systems, which can be considered both in discrete or continuous time. Discrete-time PWA systems are described by

$$
\begin{aligned}
\boldsymbol{x}(k+1) & =\boldsymbol{A}_{q} \boldsymbol{x}(k)+\boldsymbol{B}_{q} \boldsymbol{u}(k)+\boldsymbol{f}_{q} \\
\boldsymbol{y}(k) & =\boldsymbol{C}_{q} \boldsymbol{x}(k)+\boldsymbol{D}_{q} \boldsymbol{u}(k)+\boldsymbol{g}_{q}
\end{aligned} \quad \text { for }\left[\begin{array}{c}
\boldsymbol{x}(k) \\
\boldsymbol{u}(k)
\end{array}\right] \in \mathcal{C}_{q},
$$

for the operation modes $q=1, \ldots, N$, where $\mathcal{C}_{1}, \ldots, \mathcal{C}_{N}$ are convex polyhedra (i.e., given by a finite number of linear inequalities) in the input/state space with non-overlapping interiors. The variables $\boldsymbol{u}(k) \in \mathbb{R}^{m}, \boldsymbol{x}(k) \in \mathbb{R}^{n}$, and $\boldsymbol{y}(k) \in \mathbb{R}^{l}$ denote the input, state, and output, respectively, at discrete time step $k$ with $k \in \mathbb{N}$.

PWA systems have been studied extensively as they form the "simplest" extension of linear systems that can still model many nonlinear and nonsmooth processes with arbitrary accuracy and that are capable of handling some hybrid phenomena.

Many authors also study the continuous-time variant of the above model, which is given by

$$
\begin{aligned}
& \dot{\boldsymbol{x}}(t)=\boldsymbol{A}_{q} \boldsymbol{x}(t)+\boldsymbol{B}_{q} \boldsymbol{u}(t)+\boldsymbol{f}_{q} \\
& \boldsymbol{y}(t)=\boldsymbol{C}_{q} \boldsymbol{x}(t)+\boldsymbol{D}_{q} \boldsymbol{u}(t)+\boldsymbol{g}_{q}
\end{aligned} \quad \text { for }\left[\begin{array}{l}
\boldsymbol{x}(t) \\
\boldsymbol{u}(t)
\end{array}\right] \in \mathcal{C}_{q}
$$

for $q=1, \ldots, N$, where $\mathcal{C}_{1}, \ldots, \mathcal{C}_{N}$ are convex polyhedra, and the time $t$ now evolves on the real line $\mathbb{R}$. In this chapter we will mostly focus on the discretetime version as one can establish some relations to other well-known hybrid model classes, such as mixed-logical dynamical models and linear complementarity models. In the next chapters the continuous-time PWA models will play a more dominant role. 


\section{Example 1.1 Integrator system with saturation}

As a very simple example of a PWA model we can consider an integrator with upper saturation:

$$
\begin{aligned}
x(k+1) & = \begin{cases}x(k)+u(k) & \text { if } x(k)+u(k) \leqslant 1 \\
1 & \text { if } x(k)+u(k) \geqslant 1\end{cases} \\
y(k) & =x(k) .
\end{aligned}
$$

If we rewrite (1.4) as in (1.3) then we get

$$
\begin{aligned}
\mathcal{C}_{1} & =\left\{(x(k), u(k)) \in \mathbb{R}^{2} \mid x(k)+u(k) \leqslant 1\right\} \\
\mathcal{C}_{2} & =\left\{(x(k), u(k)) \in \mathbb{R}^{2} \mid x(k)+u(k) \geqslant 1\right\} \\
A_{1} & =1, \quad A_{2}=0, \quad B_{1}=1, \quad B_{2}=0 \\
f_{1} & =0, \quad f_{2}=1, \quad C_{1}=C_{2}=1 \\
D_{1} & =D_{2}=0, \quad g_{1}=g_{2}=0 .
\end{aligned}
$$

The system has two operation modes $(q=1$ and $q=2)$ for which two sets of parameter matrices are described above.

More details on PWA systems and additional results involving control of PWA systems are presented in Chapter 4 of this handbook.

\subsubsection{Mixed logical dynamical Systems}

In [Bemporad and Morari, 1999] a class of hybrid systems has been introduced in which logic, dynamics, and constraints are integrated. This resulted in the description

$$
\begin{gathered}
\boldsymbol{x}(k+1)=\boldsymbol{A} \boldsymbol{x}(k)+\boldsymbol{B}_{1} \boldsymbol{u}(k)+\boldsymbol{B}_{2} \boldsymbol{\delta}(k)+\boldsymbol{B}_{3} \boldsymbol{z}(k) \\
\boldsymbol{y}(k)=\boldsymbol{C} \boldsymbol{x}(k)+\boldsymbol{D}_{1} \boldsymbol{u}(k)+\boldsymbol{D}_{2} \boldsymbol{\delta}(k)+\boldsymbol{D}_{3} \boldsymbol{z}(k) \\
\boldsymbol{E}_{1} \boldsymbol{x}(k)+\boldsymbol{E}_{2} \boldsymbol{u}(k)+\boldsymbol{E}_{3} \boldsymbol{\delta}(k)+\boldsymbol{E}_{4} \boldsymbol{z}(k) \leqslant \boldsymbol{g}_{5},
\end{gathered}
$$

where $\boldsymbol{x}(k)$ can contain both real and boolean (i.e., 1 or 0$)$ components $(y(k)$ and $u(k)$ have a similar structure), and where $\boldsymbol{z}(k)$ and $\boldsymbol{\delta}(k)$ are respectively real-valued and boolean auxiliary variables. The inequalities $(1.5 \mathrm{c})$ have to be interpreted componentwise. Systems of the form (1.5) are called mixed logical dynamical (MLD) systems.

\subsubsection{Complementarity systems}

Complementarity systems arise when differential equations

$$
\begin{aligned}
\dot{\boldsymbol{x}}(t) & =\boldsymbol{f}(\boldsymbol{x}(t), \boldsymbol{w}(t), \boldsymbol{u}(t)) \\
\boldsymbol{z}(t) & =\boldsymbol{g}(\boldsymbol{x}(t), \boldsymbol{w}(t), \boldsymbol{u}(t))
\end{aligned}
$$


are coupled to "complementarity conditions"

$$
0 \leq \boldsymbol{z}(t) \perp \boldsymbol{w}(t) \geq 0,
$$

where the inequalities are interpreted componentwise and $\perp$ indicates the orthogonality between the vectors $\boldsymbol{z}(t)$ and $\boldsymbol{w}(t)$, i.e., $\boldsymbol{z}^{\mathrm{T}}(t) \boldsymbol{w}(t)=0$. In the above description $\boldsymbol{x}(t)$ is the state, $\boldsymbol{u}(t)$ is the control input, and $\boldsymbol{w}(t), \boldsymbol{z}(t)$ are the complementarity variables. The complementarity conditions (1.6c) constitute a particular system of equalities and inequalities, which are related to the well-known relations between the constraint variables and Lagrange multipliers in the Karush-Kuhn-Tucker conditions for optimality, the voltage-current relationship of ideal diodes, the conditions between unilateral constraints and reaction forces in constrained mechanics, etc. As such, the complementarity framework includes mechanical systems with unilateral constraints, constrained optimal control problems, switched electrical circuits, piecewise linear systems, etc.

To reveal the hybrid nature of complementarity systems, observe that (1.6c) implies that $w_{i}(t)=0$ or $z_{i}(t)=0$ for each $i \in\{1, \ldots, m\}$. As a consequence, the system (1.6) has $2^{m}$ modes. Each mode can be characterized by the active index set $J \subseteq\{1, ; m\}$, such that $z_{i}=0$, if $i \in J$, and $w_{i}=0$, if $i \in J^{\mathrm{c}}$, where $J^{\mathrm{c}}:=\{1, \ldots, n\} \backslash J$. For the mode corresponding to $J$ the dynamics is given by the following system of differential and algebraic equations (DAEs):

$$
\begin{gathered}
\dot{\boldsymbol{x}}(t)=\boldsymbol{f}(\boldsymbol{x}(t), \boldsymbol{w}(t), \boldsymbol{u}(t)) \\
\boldsymbol{z}(t)=\boldsymbol{g}(\boldsymbol{x}(t), \boldsymbol{w}(t), \boldsymbol{u}(t)) \\
z_{i}(t)=0 \text { if } i \in J \text { and } w_{i}(t)=0 \text { if } i \in J^{c},
\end{gathered}
$$

The evolution of system (1.6) will be governed by (1.7) for the mode corresponding to $J$ as long as the remaining inequalities (the "invariant" in the terminology of hybrid automata) in (1.6c)

$$
z_{i}(t) \geq 0 \text { if } i \in J^{c} \text { and } w_{i}(t) \geq 0 \text { if } i \in J
$$

are satisfied. Impending violation of (1.8) will trigger a mode change. As a consequence, during the evolution in time of the system several mode dynamics will be active successively and resets of the state vector might be necessary (think of constrained mechanical systems with impacts).

\subsubsection{Discretely controlled continuous systems}

In an important field of application of switched systems the switching signal is determined by a controller in dependence on the state $\boldsymbol{x}$. The structure of discretely controlled continuous systems is shown in Fig. 1.3. The control input $q(t)$ is a discrete signal that prescribes the operation mode of the plant. 


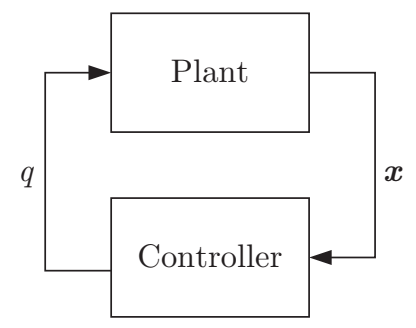

Fig. 1.3. Discretely controlled continuous system
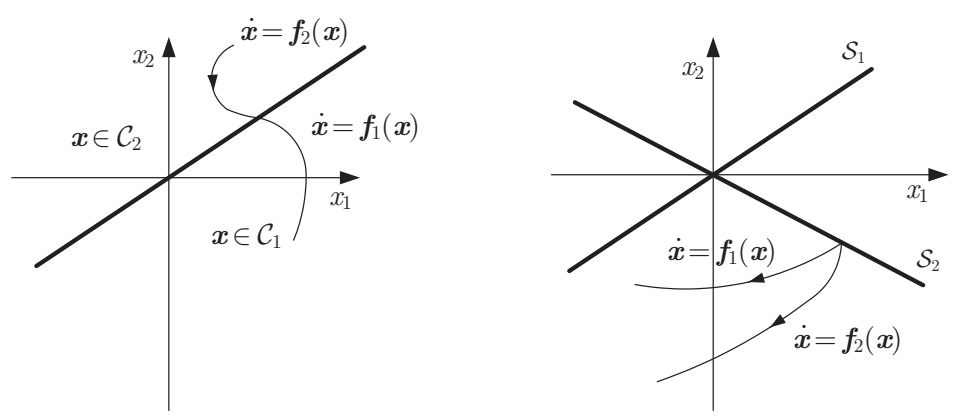

Fig. 1.4. Comparison of the switching schemes of piecewise affine and discretely controlled continuous system

The difference between the switching schemes used in PWA or discretely controlled continuous systems is illustrated in Fig. 1.4. For PWA systems, the continuous state space $\mathbb{R}^{n}$ is partitioned into sets $\mathcal{C}_{q}(q \in \mathcal{Q})$ and the $q$ th vector field $\boldsymbol{f}_{q}$ is valid as long as the state $\boldsymbol{x}$ remains in the set $\mathcal{C}_{q}$. Hence, the operation mode $q(t)$ at time $t$ depends only on the current continuous state $\boldsymbol{x}(t)$. In the figure, the trajectory $\boldsymbol{x}($.$) first goes through the partition \mathcal{C}_{2}$ and, hence, the vector field $\boldsymbol{f}_{2}$ is valid. After crossing the boundary between the sets $\mathcal{C}_{2}$ and $\mathcal{C}_{1}$, the future state trajectory is governed by the vector field $\boldsymbol{f}_{1}$.

Contrary to this, discretely controlled continuous systems use a switching scheme that is usually described by switching surfaces $\mathcal{S}_{i}$, which are hyperplanes in the state space $\mathbb{R}^{n}$. If the state trajectory $\boldsymbol{x}($.$) crosses such a surface$ at time $\bar{t}$, the discrete state is changed $\left(q \rightarrow q^{\prime}\right)$, where the successor state is determined by the switching surface touched at time $\bar{t}$. Whether the trajectory crosses the surface $\mathcal{S}_{i}$ or not depends on the vector field that becomes active at time $\bar{t}$. In the figure, the new vector field $\boldsymbol{f}_{2}$ moves the state back into the region from which it came. In the region "below" the surfaces $\mathcal{S}_{1}$ and $\mathcal{S}_{2}$ the state trajectory is first governed by $\boldsymbol{f}_{1}$ and later by $\boldsymbol{f}_{2}$. Hence, the current discrete state $q$ depends on the whole trajectory $\boldsymbol{x}($.$) and not only on$ the current state $\boldsymbol{x}(t)$. It is a "true" discrete state. 


\subsubsection{Timed automata}

Timed automata are a class of hybrid automata that involve particularly simple continuous dynamics: all differential equations are of the form $\dot{x}=1$, and all the invariants, guards, etc. involve comparison of the real-valued states with constants (e.g., $x=1, x<2, x \geq 0$, etc.). Clearly, timed automata are somewhat limited when it comes to modeling physical systems. They are very suited, however, for encoding timing constraints (such as "event A must take place at least 2 seconds after event B and not more than 5 seconds before event C", etc.). For some applications, such as multimedia, internet, and audio protocol verification, this type of description is sufficient for both the dynamics of the system and the properties that we want the system to satisfy. Readers interested in the details of timed automata are referred to [Alur and Dill, 1994].

\subsubsection{Hybrid inclusions}

Hybrid inclusions form a natural extension of differential inclusions $\dot{\boldsymbol{x}} \in F(\boldsymbol{x})$ in the sense that invariants, guards, and resets are added. Hybrid inclusions are given by the data of two subsets $\mathcal{C}$ (the flow set) and $\mathcal{D}$ (the jump set) of $\mathbb{R}^{n}$, and two set-valued mappings $\boldsymbol{F}: \mathcal{C} \rightarrow \mathbb{R}^{n}$ and $\boldsymbol{G}: \mathcal{D} \rightarrow \mathbb{R}^{n}$. The hybrid inclusion is then written as

$$
\begin{aligned}
\dot{\boldsymbol{x}} \in \boldsymbol{F}(\boldsymbol{x}) & \text { if } \boldsymbol{x} \in \mathcal{C} \\
\boldsymbol{x}^{+} \in \boldsymbol{G}(\boldsymbol{x}) & \text { if } \boldsymbol{x} \in \mathcal{D} .
\end{aligned}
$$

Clearly, this description provides compact models with a clear structure, which encompasses many hybrid phenomena. The hybrid inclusions turned out to be useful, e.g., in the general study of hybrid systems and in the domains of networked control systems and reset control systems in particular.

\subsection{Comparison of the models}

\subsubsection{Equivalence of model classes}

It is of interest to know which type of model classes are equivalent or which model classes encompass others, as this can assist in transforming analysis and synthesis results and tools obtained for one class to another.

In [Heemels et al., 2001] relationships between some classes of discrete-time hybrid models are presented, which will be summarized in Section 5.3 of this handbook. There it will be shown that under mild assumptions discrete-time PWA systems are equivalent to some other classes of hybrid systems such as MLD systems and discrete-time linear complementarity systems. Specific analysis or design problems might be easier to solve using the formulation of 
one of the subclasses than that of another subclass. As a result, each system class has its own analysis and synthesis tools. As a consequence, it really depends on the problem and application at hand, which of these classes is best suited.

In the continuous-time framework, such broad equivalence relations are out of the question for the mentioned classes. However, there are relations between linear complementarity systems and other specific classes of non-smooth systems such as the "normal cone differential inclusions" and projected dynamical systems [Brogliato et al., 2006; Heemels et al., 2000a].

Also for many simulation and verification tools it is of interest to transform model structures into others. The interchange format that is discussed in Chapter 12 of this handbook forms the means to transform models into certain basic formats, which can be used for various simulators and model checkers.

\subsubsection{Modeling power versus decisive power}

The choice of a suitable modeling framework is a trade-off between two conflicting criteria: the modeling power and the decisive power. The modeling power indicates the size of the class of systems allowing a reformulation in terms of the chosen model description. The decisive power is the ability to prove quantitative and qualitative properties of individual systems in the framework. A model structure that is too broad (like the hybrid automaton) cannot reveal specific properties of a particular element in the model class. The size of a model class is often taken too large for analysis purposes. As indicated by [Blondel and Tsitsiklis, 1999], even for the easiest hybrid systems analysis and control problems are often undecidable, which means that, roughly speaking, there does not exist an algorithm that solves the problem and for which finite termination can be guaranteed. Even if the problems are decidable, then often the problems require a very high computational load to be solved (NP-complete or NP-hard, see [Garey and Johnson, 1979] for details on these terms). Also for many classes of hybrid and timed automata the reachability problem (i.e., determining whether some of the trajectories of the system can attain a specific set of desired states) is undecidable [Henzinger et al., 1998].

\section{Example 1.2 NP-hard analysis problem}

As an example of an NP-hard problem, [Blondel and Tsitsiklis, 1999] considers the elementary hybrid system given by

$$
\boldsymbol{x}(k+1)= \begin{cases}\boldsymbol{A}_{1} \boldsymbol{x}(k) & \text { if } \boldsymbol{c}^{\mathrm{T}} \boldsymbol{x}(k) \geq 0 \\ \boldsymbol{A}_{2} \boldsymbol{x}(k) & \text { if } \boldsymbol{c}^{\mathrm{T}} \boldsymbol{x}(k)<0\end{cases}
$$

where $\boldsymbol{A}_{1}, \boldsymbol{A}_{2}$ are matrices and $\boldsymbol{c}$ is a (column) vector of appropriate dimensions. The problem of deciding whether this switching system is stable is shown to be 
NP-hard. Loosely speaking, this means that there is no algorithm that answers the question of stability in polynomial time (as function of the size of $\boldsymbol{A}_{1}, \boldsymbol{A}_{2}$, and $\boldsymbol{c}$ ).

As a conclusion, one can state that in certain cases it is useful to have some additional structure on the model class that one considers as the hybrid automaton model is too broad for detailed analysis.

\subsection{Problems resulting from hybrid phenomena}

Hybrid systems are inherently nonlinear and non-smooth, and therefore many of the results available from the vast literature on linear systems and smooth nonlinear systems do not apply. As a consequence, many basic systemtheoretic problems like well-posedness, stability, controllability, observability, safety, etc. and many design methods for controllers and observers have to be reconsidered within the hybrid context. Next, we will discuss several particular problems that complicate the resolution of these issues.

\subsubsection{Inadequacy of mono-disciplinary approaches}

Hybrid system already exist for a long time and engineers have managed to analyze and to design them. So, how did they manage to do so and what is new in hybrid systems theory?
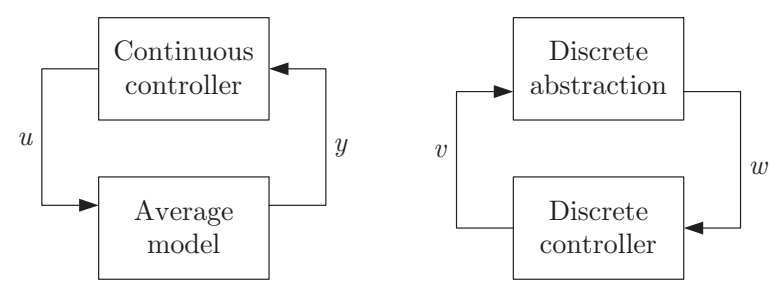

Fig. 1.5. Two ways of simplifying hybrid systems analysis and design

Figure 1.5 shows two ways to deal with hybrid systems by avoiding the combination of continuous and discrete model parts. These approaches either abstract from the discrete state evolution and end up with a purely continuous representation, or abstract from the continuous movement and represent the system by a discrete-event model. In both cases, either the methods elaborated in the theory of continuous systems or the theory of discrete-event systems alone are sufficient for analysis and design.

As an example of the approach presented on the left-hand side of Fig. 1.5, consider a DC-DC converter. A discrete controller switches the operation mode in a very high frequency (usually measured in $\mathrm{MHz}$ ), so that the voltage 
control problem can be solved by using an average model that describes the continuous evolution of an average state approximating the highly oscillating voltage.

On the other hand, if only the discrete-event behavior of the system is important, then the continuous state evolution can be ignored or reduced to its influence on the discrete state changes. The model that represents the discrete state transitions of a hybrid system is called a discrete abstraction.

In both situations, the hybrid character of the system under consideration is ignored (or "abstracted away"). This may be reasonable under specific practical circumstances, because any model should not represent a system in the best possible way, but in a way suitable for solving the given task. However, the important consequence of the abstraction is the fact that typical phenomena of hybrid systems such as switching dynamics and state jumps can no longer be represented by the model and are, thus, excluded from the analysis and control design.

The main aim of hybrid systems theory is to elaborate analysis and design methods for technological systems for which both the continuous and the discrete state evolution play an important role and for which neither an abstraction from the continuous movement nor the exclusion of the discrete state transitions from the considerations is possible or suitable, and for which the interaction between both system parts thus has to be taken into account.

The following subsections describe phenomena that cannot be adequately modeled and analyzed by analysis methods purely tailored for continuous systems or for discrete-event systems. So methods for both system classes have to be combined in a suitable manner to deal with these phenomena.

\subsubsection{Instability of hybrid systems resulting from switching}

The fact that hybrid techniques have to be developed is evidenced by the study of stability of hybrid systems. Stability is a real "hybrid problem," that cannot be tackled by studying, e.g., the stability of the subsystems only (except for some trivial examples). This is illustrated by the following switched system taken from [Branicky, 1998].

\section{Example 1.3 Unstable hybrid system with stable operation modes}

Consider the hybrid system with two operation modes

$$
\dot{\boldsymbol{x}}= \begin{cases}\boldsymbol{A}_{1} \boldsymbol{x} & \text { if } x_{1} x_{2} \leq 0 \\ \boldsymbol{A}_{2} \boldsymbol{x} & \text { if } x_{1} x_{2}>0\end{cases}
$$

with

$$
\boldsymbol{A}_{1}=\left(\begin{array}{cc}
-1 & 10 \\
-100 & -1
\end{array}\right) \text { and } \boldsymbol{A}_{2}=\left(\begin{array}{cc}
-1 & 100 \\
-10 & -1
\end{array}\right)
$$


By inspection of the eigenvalues of $\boldsymbol{A}_{1}$ and $\boldsymbol{A}_{2}$, which for both matrices are $\lambda_{1 / 2} \approx$ $-1 \pm 31.62 \mathrm{j}$, one can see that both of the dynamics are stable (cf. the phase portraits in Fig. 1.6), but the switched system (1.9) is not (see Fig. 1.7).
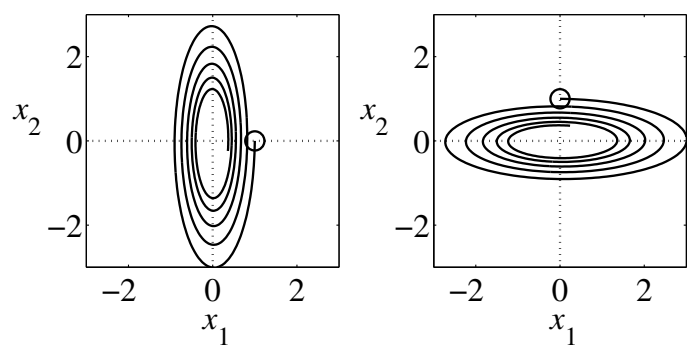

Fig. 1.6. Behavior of the stable linear submodels

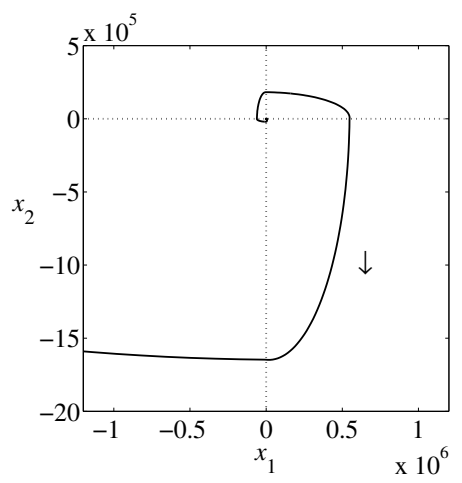

Fig. 1.7. Unstable switched system (1.9).

Indeed, the switched system activates dynamics 1 if the state lies in the second and the fourth quadrant, and dynamics 2 if the state is in the first or the third quadrant. As one can see from the trajectory shown in Fig. 1.7, the switched system is unstable.

Also converse examples exist where the subsystems are all unstable and the corresponding switched system is stable (e.g., consider the time-reversed version of the system above).

As a consequence, it is not sufficient to study stability properties of the subsystems only, as the switching structure has to be taken into account as well. For many other properties (observability, controllability, etc.), this is also the case and hence, hinging on the available results for continuous and discrete-event models, a hybrid systems theory is needed. 


\subsubsection{Zeno behavior}

The evolution of a hybrid system typically consists of smooth phases in which the discrete mode remains constant, separated by discrete events and actions. In the terminology of hybrid automata, the discrete events are often given by guards being enabled or invariants impending to be violated and the discrete actions are mode switches and/or resets of the continuous part of the hybrid state variable.

Zeno behavior is the phenomenon that for a dynamical system an infinite number of events occur in a finite length time-interval.

This phenomenon is named after the ancient Greek philosopher Zeno of Elea.

\section{Example 1.4 Bouncing ball}

A famous example of a simple model of a mechanical system that exhibits Zeno behavior is the bouncing ball (Fig. 1.8).
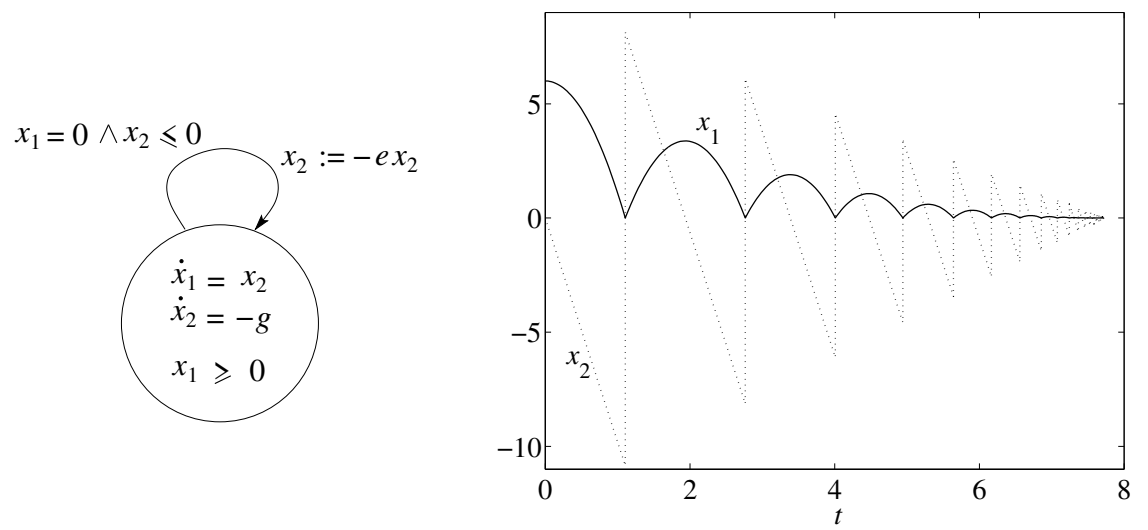

Fig. 1.8. Bouncing ball: hybrid automaton (left) and simulation (right) with $x=x_{1}$ and $\dot{x}=x_{2}$.

The height of the ball above the surface is denoted by $x_{1}$ with dynamics $\ddot{x}_{1}=$ $-g$ and the constraint $x_{1} \geq 0$. The velocity $\dot{x}_{1}$ of the ball will be denoted by $x_{2}$. To complete the model we include Newton's restitution rule $x_{2}(\tau+)=-\alpha x_{2}(\tau-)$ when $x_{1}(\tau-)=0$ and $x_{1}(\tau-)<0(0<\alpha<1)$. The reset or event times $\left\{\tau_{i}\right\}_{i \in \mathbb{N}}$ can be easily computed and are given by

$$
\tau_{i+1}=\tau_{i}+\frac{2 \alpha^{i} x_{2}(0)}{g}, \quad \text { for } i \in \mathbb{N}
$$

assuming that $x_{1}(0)=0$ and $x_{2}(0)>0$. Hence, $\left\{\tau_{i}\right\}_{i \in \mathbb{N}}$ has a finite limit equal to $\tau^{*}=\frac{2 x_{2}(0)}{g-g \alpha}<\infty$. So the continuous state $\left(x_{1}(t), x_{2}(t)\right)$ converges to $(0,0)$ when $t \uparrow$ 
$\tau^{*}$. The physical interpretation is that the ball is at rest within a finite time span, but after infinitely many bounces. By extending the model (the complementarity framework presented above is quite natural for this) a continuation beyond $\tau^{*}$ can be defined by $\left(x_{1}(t), x_{2}(t)\right)=(0,0)$ for $t>\tau^{*}$.

This is a specific example of Zeno behavior, i.e., a infinite number of events in a finite length time interval. In this case we have an infinite number of state reinitializations and the set of event times for the bouncing ball contains a so-called right-accumulation point.

This kind of Zeno behavior often prevents the existence of global solutions (i.e., defined for all times $t \in \mathbb{R}_{+}$) and hence, is directly related to the wellposedness issue: the existence and uniqueness of solutions given an initial condition. The definition of a solution trajectory can either allow for specific kind of Zenoness or not. Of course, depending on the choice of solutions, the well-posedness issue differs.

Also for the simulation of hybrid systems, Zenoness is a difficult problem. Imagine that one tries to determine a trajectory of a system like the bouncing ball by iteratively integrating over the smooth phases, estimating the event times (by detecting zero crossings), resetting the states, etc. Of course, this natural integration scheme (sometimes called "event-driven integration") would get stuck at or before the right-accumulation point as the interval lengths are decreasing to arbitrary small positive numbers. Some limiting procedure would be necessary to determine the accumulation point, which would be numerically hard to detect and to implement. Also the detection of zero crossings is crucial (as they determine the next mode for instance). As numerical simulators always produce approximation errors, the fact that hybrid systems do not generally have the property of continuous dependence on initial conditions (see also Section 1.3.5 below), the slightest error might lead to approximating a completely different trajectory. For certain specific classes of hybrid systems, so-called time-stepping schemes [Camlibel et al., 2002; Acary and Brogliato, 2008] might be more suitable.

Not only for well-posedness or simulation issues, but also for analysis one has to be careful as certain properties of a system may only be true for trajectories that are defined on finite-length time intervals due to Zeno behavior. This might imply that the property does not hold true for all times (beyond the Zeno point). As a consequence, in case of verification of certain system properties, it is crucial which type of trajectories to include in the model of the plant (and hence, in the analysis). Definitely the mathematical behavior of the model should be "rich enough" to reflect the real plant or system behavior. What one often sees, is that, e.g., Zeno solutions are excluded in the analysis of system's properties. In this case one has to realize that the property holds only for "non-Zeno" trajectories, while the actual system might have solutions beyond Zeno points and the system might fail in practice. One (in)famous example consisting of two tanks is given in [Alur and Henzinger, 1993]. There a controller is designed that keeps the fluid level for both tanks above a desired minimal level and this is proven to work for non-Zeno trajectories. As 
the (real) system has Zeno trajectories, this analysis is useless and it turns out that both tanks do get below the minimal level. Hence, this demonstrates that one has to be careful with excluding these type of phenomena, or stated differently, with the choice of solution concept used for the analysis.

The above aspects indicate that the Zeno phenomenon is a major problem in hybrid systems analysis and it is one of the challenges that the system and control theory for hybrid systems has to face. Hence, it is important to develop conditions that either exclude Zenoness or indicate when it is present. As the Zeno behavior can be seen as a modeling artifact that never can occur in reality, these conditions should be used to avoid Zeno behavior of the model.

\subsubsection{Chattering or infinitely fast switching: sliding modes}

For hybrid systems in general and the switched systems described in Section 1.1.3 in particular, also the presence of infinitely fast switching resulting in sliding behavior around switching surfaces can complicate the analysis. As Fig. 1.9 shows, chattering occurs if the vector fields $\boldsymbol{f}_{-}$and $\boldsymbol{f}_{+}$that hold on either side of the surface $\mathcal{S}$ both point to the switching surface. Hence, the trajectory is forced to remain on $\mathcal{S}$. Consequently, the surface is called a sliding surface. In practice, however, the state will not remain on this surface, but a fast switching will occur (that may overload the actuators).

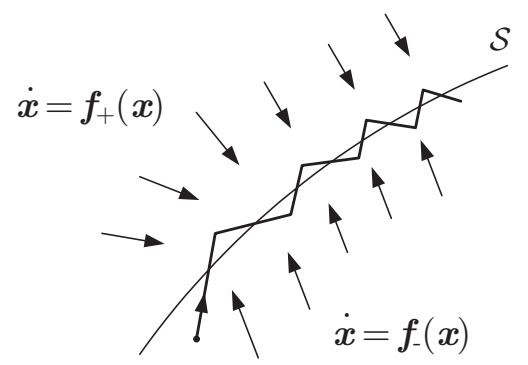

Fig. 1.9. Chattering and sliding modes

Properties that might be true for the constituting dynamics do not necessarily hold for the sliding modes induced by these dynamics. For instance, two stable systems might yield an unstable sliding mode (even if there is a continuous piecewise quadratic Lyapunov function corresponding to each of the individual dynamics), as was indicated by Example 2.3 in [Liberzon, 2003] and is evidenced by the following example.

Example 1.5 Chattering of piecewise linear system

Consider the piecewise linear system (taken from [Heemels and Weiland, 2008]) 


$$
\dot{\boldsymbol{x}}=\left\{\begin{array}{ll}
\boldsymbol{A}_{1} x & \text { if } \boldsymbol{x}_{1} \geq 0 \\
\boldsymbol{A}_{2} x & \text { if } \boldsymbol{x}_{1} \leq 0
\end{array} \quad \text { with } \boldsymbol{A}_{1}=\left(\begin{array}{ll}
-3 & 1 \\
-5 & 1
\end{array}\right), \boldsymbol{A}_{2}=\left(\begin{array}{cc}
-3 & -1 \\
5 & 1
\end{array}\right)\right.
$$

This system allows a continuous piecewise quadratic Lyapunov function of the form $V(\boldsymbol{x})=\boldsymbol{x}^{\mathrm{T}} \boldsymbol{P}_{1} \boldsymbol{x}$ when $\boldsymbol{x}_{1} \geq 0$ and $V(\boldsymbol{x})=\boldsymbol{x}^{\mathrm{T}} \boldsymbol{P}_{2} \boldsymbol{x}$ when $x_{1} \leq 0$ with

$$
\boldsymbol{P}_{1}=\left(\begin{array}{cc}
3.9140 & -2.0465 \\
-2.0465 & 1.5761
\end{array}\right) \text { and } \boldsymbol{P}_{2}=\left(\begin{array}{ll}
3.9140 & 2.0465 \\
2.0465 & 1.5761
\end{array}\right)
$$

which are computed via the procedure outlined in [Johansson and Rantzer, 1998]. These results prove the exponential stability of the system along "ordinary" solutions (without sliding motions).

However, the sliding mode dynamics at $x_{1}=0$ is given by $\dot{x}_{2}=x_{2}$, which is unstable, in spite of the presence of a continuous piecewise quadratic Lyapunov function (satisfying $\boldsymbol{A}_{q}^{\mathrm{T}} \boldsymbol{P}_{q}+\boldsymbol{P}_{q} \boldsymbol{A}_{q}<0$ and $\boldsymbol{P}_{q}>0$ for $q=1,2$ ).

Hence, the presence of possible sliding motions has to be detected and included in a proper manner in analysis or synthesis methods.

\subsubsection{Sensitivity and nondeterminism of the system behavior}

The qualitative behavior of hybrid systems can be very sensitive to changes in the continuous initial state. As a consequence, the discrete behavior, which is represented by the sequence of discrete states, can be nondeterministic in the sense that for a small change in the initial continuous state $\boldsymbol{x}_{0}$ it is unknown whether the discrete state sequence remains the same or changes drastically.
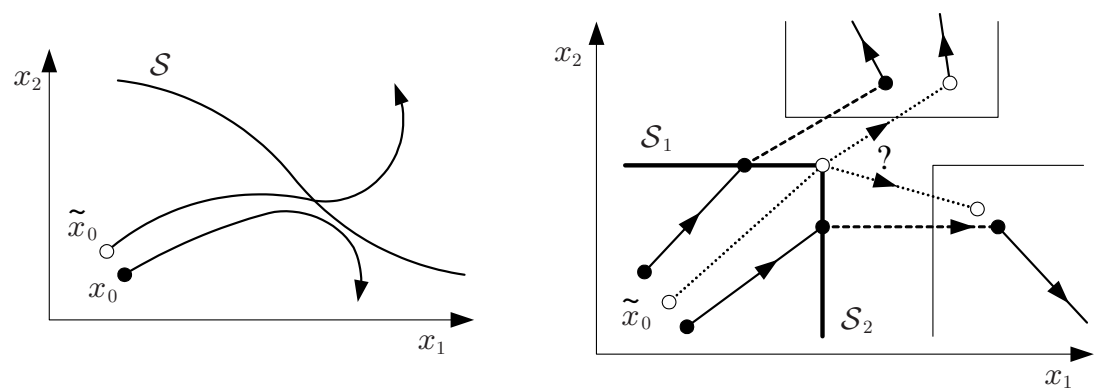

Fig. 1.10. Sensitivity of the hybrid system behavior

The reason for this is illustrated in Fig. 1.10. The left part of the figure shows two continuous state trajectories that start in nearby initial states $\boldsymbol{x}_{0}$. As one trajectory crosses the switching surface $\mathcal{S}$ whereas the other does not, the continuations of the continuous state trajectories differ considerably. In particular, the hybrid system changes its discrete state in the first case, whereas it remains in the current discrete state in the second case. Note that for any given switching surface one can in general find a point in which the 
surface is tangential to the vector field. Consequently, the phenomenon shown here is not specific for this example, but occurs in many hybrid systems.

Another situation, where this sensitivity becomes obvious, is illustrated in the right part of the figure, which shows state trajectories with state jumps. The jumps are invoked when the continuous state trajectory crosses one of the switching surfaces $\mathcal{S}_{1}$ or $\mathcal{S}_{2}$. As the reset map $\mathcal{R}$ associates quite different state sets to the jumps that start at $\mathcal{S}_{1}$ or at $\mathcal{S}_{2}$, respectively, the continuation of the continuous and the discrete state trajectories depends severely on the fact which switching surface has been reached. Furthermore, there is an initial state $\boldsymbol{x}_{0}$ for which the continuation of the behavior after the state jump cannot be unambiguously predicted (at least if the usual model uncertainties are taken into account, cf. dotted line in the figure).

\subsection{Overview of solution approaches}

\subsubsection{Necessity for a new hybrid systems theory}

In this section we survey the main approaches that have been developed to deal with the new phenomena occurring in hybrid systems. The consequences for control system analysis and design are also explained.

A first look at hybrid systems raises a very basic question:

Does the class of hybrid systems necessitate a new theory?

This question is reasonable, because a mixture of continuous and discrete effects have been dealt with in control engineering for long. Think, in particular, of optimal control, where a time-optimal solution represents a "bangbang" type control input or where inequality constraints can switch between an active and an inactive status. In control practice, gain scheduling methods or fuzzy control are used with some success and these methods are based on switching among different control laws in dependence upon the current operating conditions. Finally, programming logic controllers often use binary or multi-valued signals to switch between continuous processes.

The main difference between these methods and hybrid systems theory lies in the fact that the traditional methods mentioned above deal with specific control aspects where the co-existence of continuous and discrete dynamics can be tackled by specific analytical methods, whereas hybrid systems theory tries to elaborate methods that allow to introduce combined continuous-discrete phenomena in a quite more general sense. The new theory starts with hybrid phenomena and aims at creating modeling formalisms and analysis methods that allow such phenomena to occur in arbitrary combinations.

The result of this approach is twofold. On the one hand, a very general class of hybrid systems can be dealt by the models that will be introduced in the following chapters. This class does not only include continuous models that are equipped with some discrete elements like inequality constraints, or 
discrete-event models that have been extended by some continuous properties like clocks. This system class is general enough to cover systems with intrinsic continuous and discrete phenomena. In particular, state jumps or vector fields with discontinuities cannot be dealt with by continuous systems theory or the methods mentioned above, which start from the basic assumption of a (Lipschitz) continuous vector field. For them, a new theory has to be elaborated.

On the other hand, as the price for this general approach, the modeling and design problems become very complex with many problems proved to be undecidable or at least NP-hard. Even if the problems are classified as "efficiently solvable" the computational complexity of real-world applications often exceeds the thresholds of the available computing resources. Hence, the generality of the models has to be reduced systematically to end up with really applicable methods.

The difference between the classical control theory and its extensions and the more general approach of hybrid systems theory becomes obvious by the new problems explained above. Phenomena like Zeno behavior, instability occurring from stable systems, sensitivity and nondeterminism of the system behavior etc. cannot be detected without using this general approach. Furthermore new challenges include the creation of reliable numerical methods for the reachability analysis and simulation of hybrid systems, the elaboration of verification methods for mixed continuously-discretely controlled systems or design procedures for event generators, and interfaces between continuous and discrete subsystems. These and further problems necessitate a new theory and the following chapters will show how far this theory is already developed.

\subsubsection{Solution concepts and well-posedness}

The new phenomena occurring in hybrid systems necessitate to define what a solution of the hybrid system is. Different solution concepts have been defined for the various model formats proposed, and even for a given model several solution concepts can be applied. The discussion at the end of the section on Zeno behavior above has already indicated the importance of selecting a suitable solution concept for the system and for the analysis or design problem at hand. Studying a two-tank system with non-Zeno type of solutions might lead to the erroneous conclusion that the fluid levels in the tanks stay above specified target values, which is not the case in reality. Also the exclusion of specific solutions, e.g., by proving that Zeno behavior cannot occur in a system, might be beneficial for subsequent analysis.

In general a fundamental problem of interest is the well-posedness property of a system.

A system is said to be well-posed if a solution of the system exists and is unique given an initial condition (and possibly input signals). 
The well-posedness property indicates that the system does not exhibit deadlock behavior (no solutions from certain initial conditions) and that determinism (uniqueness of solutions) is satisfied. Especially, when the hybrid models represent physical systems well-posedness is of interest. For certain other hybrid systems, actually the determinism might be a too strong requirement and one might only focus on the existence of solutions given initial conditions and inputs.

\subsubsection{Controllability, observability, and stability}

Notions such as controllability, stabilizability, observability and detectability have played a central role throughout the history of modern control theory. Conceived by KALMAN, the controllability concept has been studied extensively in the context of finite-dimensional linear systems, nonlinear systems, infinite-dimensional systems, hybrid systems, and behavioral systems. One may refer to, e.g., SonTAG's book [Sontag, 1998] for historical comments and references.

Outside the linear context, characterizations of global controllability have been hard to obtain. In the setting of smooth nonlinear systems, results have been obtained for local controllability, but there is no hope to obtain general algebraic characterizations of controllability in the large. The complexity of characterizing controllability and stabilizability has been studied in [Blondel and Tsitsiklis, 1999] for some classes of hybrid systems, and the authors show that even within quite limited classes there is no algorithm to decide the controllability status of a given system. Hence, this indicates that there is no hope to find complete conditions for general hybrid systems. The best to obtain seems to be characterizations for some specific classes of hybrid systems.

Controllability problems for piecewise linear systems and various related model classes have been studied in [Lee and Arapostathis, 1987; Bemporad et al., 2000b; Blondel and Tsitsiklis, 1999; Habets and van Schuppen, 2001; Camlibel et al., 2008]. A similar story holds for observability and detectability [Bemporad et al., 2000b; Camlibel et al., 2006; Babaali and Egerstedt, 2004; Collins and van Schuppen, 2004].

It has already been indicated that stability for hybrid systems is a very complex problem. For some classes of hybrid systems such as switched systems and PWA systems methods have been developed to analyze stability through various types of Lyapunov functions such as common quadratic Lyapunov functions, continuous piecewise quadratic Lyapunov functions, discontinuous piecewise quadratic Lyapunov functions, etc. These and other stability results for hybrid systems are presented in [Ye et al., 1998; Liberzon and Morse, 1999; Liberzon, 2003; Johansson and Rantzer, 1998; Mignone et al., 2000; Chen et al., 2004; Rantzer and Johansson, 2000]. 


\subsubsection{Control design}

Several control methods have been developed for hybrid systems, or more specifically, for special classes of hybrid systems.

For switched systems a wide body of literature exists on the development of stabilizing controllers using Lyapunov arguments and linear matrix inequalities (cf. [Wicks and DeCarlo, 1997; Johansson, 2003; Mignone et al., 2000]).

For a class of hybrid systems appearing in the context of manufacturing a control approach based on optimal control has been developed in [Cassandras et al., 2001; Cho et al., 2001; Pepyne and Cassandras, 1998].

Another important stream of control results for hybrid systems involves model predictive control (MPC). MPC has originally been developed for linear systems in the pioneering work by Richalet, Cutler, and Ramaker [Richalet et al., 1978; Cutler and Ramaker, 1979]. Recently it has also been extended to some classes of hybrid systems. MPC uses (on-line) optimization in combination with a prediction model and a receding horizon approach to determine control inputs that optimize the performance of the system over a given prediction horizon subject to various operational and functional constraints on the inputs, states, and outputs of the system.

In the seminal paper [Bemporad and Morari, 1999] an MPC approach is presented for mixed logical dynamical (MLD) systems and thus also for PWA systems due to existing equivalence relations mentioned before. Additional results on MPC for MLD and PWA systems can be found in [Bemporad et al., 2000a; Mayne and Raković, 2003; Lazar et al., 2006; Borrelli, 2003]. For related classes of hybrid systems MPC approaches have been developed in [De Schutter and van den Boom, 2004; Necoara et al., 2006].

\subsubsection{Observer design}

Observer design for hybrid systems is also a topic of interest. In many practical situations the full state is not available for feedback, while most control design methods mentioned before are based on state feedback. As such, it is of interest to use output feedback controllers using, e.g., a "certainty equivalence principle" in which the state feedback will be based on an estimated state coming from an observer or another estimation scheme.

Several interesting papers are available on observer design for hybrid systems, especially in the context of switched and piecewise linear systems. Nevertheless, the problem is still of interest as it turns out to be rather complicated, especially when the mode $q$ of the system is not known or cannot be directly reconstructed on the basis of the measurements. This situation occurs, for example, if only the continuous input $\boldsymbol{u}(t)$ and continuous output $\boldsymbol{y}(t)$ are measurable and the discrete state $q$ has to be estimated by means of this information (Fig. 1.11).

The situation becomes even more complicated when resets of the continuous state variable occur. A good starting point for investigating hybrid 


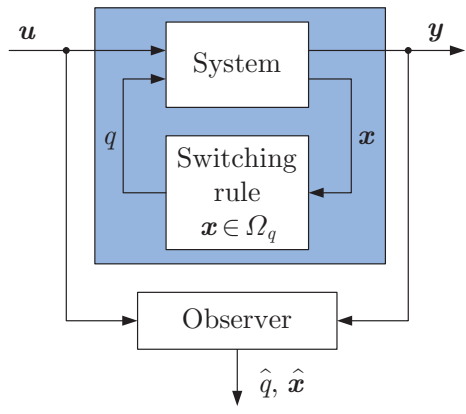

Fig. 1.11. State observation of hybrid systems

observer design are the following references: [Alessandri and Coletta, 2001; Bara et al., 2001; Petterson, 2005; Juloski et al., 2007; Balluchi et al., 2002; Pavlov et al., 2005; Ferrari-Trecate et al., 2002].

\subsubsection{Identification}

Models of hybrid systems typically contain parameters that cannot always be determined using a first-principles modeling approach. In such cases the parameters have to be determined based on input-output data. This process is called identification. Some results on identification of hybrid systems are described in [Ferrari-Trecate et al., 2003; Roll et al., 2004; Vidal et al., 2003; Juloski et al., 2005b; Bemporad et al., 2005]. A comparison between various identification procedures is given in [Juloski et al., 2005a]. This comparison paper also presents interesting problems that hybrid identification approaches have to face.

\subsubsection{Model checking and verification}

The process of automatically analyzing the properties of systems by exploring their state space is known as model checking. This problem is only decidable for a few classes of hybrid systems, among which timed automata [Alur et al., 2000; Henzinger et al., 1998].

Timed automata were the first class of hybrid systems that were shown to be amenable to model checking methods [Alur and Dill, 1994]. Since then a number of other classes of hybrid systems with this property have been established: classes of multi-rate automata [Alur et al., 1995], classes of systems with continuous dynamics governed by constant differential inclusions [Henzinger et al., 1995], and classes of systems with continuous dynamics governed by linear differential equations [Lafferriere et al., 2000]. It has also been shown that a very wide class of hybrid systems can be approximated arbitrarily closely by such "decidable" hybrid systems [Puri et al., 1995] (albeit 
at the cost of exponential computational complexity). For overview of the developments in this area see [Alur et al., 2000].

\subsubsection{Robust stability}

To indicate some of the robustness problems that can occur in hybrid systems, let us consider the following example taken from [Kellett and Teel, 2004]:

$$
x(k+1)= \begin{cases}0, & \text { if } x(k) \leq 1 \\ 1, & \text { if } x(k)>1 .\end{cases}
$$

This system is exponentially stable (actually every trajectory converges to the origin within 2 steps). However, the system has no robustness in the sense that the perturbed system

$$
x(k+1)= \begin{cases}w(k), & \text { if } x(k) \leq 1 \\ 1+w(k), & \text { if } x(k)>1\end{cases}
$$

has solutions with $|x(k)| \geq 1$ no matter how small the bound $\varepsilon$ on the disturbances $|w(k)| \leq \varepsilon$ is taken, indicating that robust stability is not present. It is well-known that for linear and smooth systems such a situation cannot arise.

In [Prieur et al., 2007] it is precisely proved that, under some regularity assumptions, the stability of hybrid systems is robust with respect to sufficiently small vanishing perturbations. This fact reflects what has long been appreciated for continuous time systems (cf. [Clarke et al., 1998] and [Ledyaev and Sontag, 1999]).

This "robustness for free" result is particularly important in the context of the design of hybrid stabilizers for nonlinear systems since in [Prieur et al., 2007] it is claimed that any stabilizing hybrid feedback, with a discrete logic variable, meeting some basic regularity assumptions is robust, even if the logic variable does not converge to a finite set. This is a general result addressing robustness of such hybrid feedbacks; in [Hespanha and Morse, 1999; Hespanha et al., 1999; Morin and Samson, 2000; Prieur and Astolfi, 2003; Prieur, 2005; Prieur and Trélat, 2006], robustness was established for particular hybrid feedbacks.

\subsubsection{Simulation}

Finally, note that in practice the most widely used technique for hybrid systems is computer simulation, via a combination of discrete-event simulation and differential algebraic equation (DAE) solvers. Some computer simulation and verification tools that are (also) used for hybrid systems are BaSiP, Modelica, HyTech, KRONOS, Hybrid Chi, 20-sim, and UPPAAL. Simulation models can represent the plant with a high degree of detail, providing a close correspondence between simulated behavior and real plant behavior. This approach 
is, for any large system, computationally very demanding, and moreover it is difficult to understand from a simulation how the behavior depends on model parameters. This difficulty is even more pronounced in the case of large-scale hybrid systems that consist of many interacting modules. Fast simulation techniques based on variance reduction, and perturbation analysis techniques [Cassandras, 1993] have been developed in order to partially overcome these limitations.

\section{Bibliographical notes}

References that can be used as a starting point of a study of hybrid system models are for hybrid automata [Branicky et al., 1998; Lynch et al., 1996], for switched systems [Liberzon, 2003], for piecewise linear or piecewise affine models [Sontag, 1981, 1996], for timed automata [Alur and Dill, 1994][Bengtsson and Yi, 2004], for the duration calculus [Chaochen et al., 1991], for timed or hybrid Petri nets [David and Alla, 1994; Holloway et al., 1997; Stremersch and Boel, 1998], for differential automata [Prieur, 2005; Tavernini, 1987], for mixed logical dynamic models [Bemporad and Morari, 1999], for real-time temporal logics [Alur and Henzinger, 1993; Pnueli, 1977], for timed communicating sequential processes [Davies, 1993; Hoare, 1985], for complementarity systems [Heemels et al., 2000b; van der Schaft and Schumacher, 1998, 1996], for hybrid inclusions [Cai et al., 2007; Goebel and Teel, 2006], for the max-min-plus scaling (MMPS) systems [De Schutter, 2000], for stochastic hybrid models [Hu et al., 2000; Pola et al., 2003], and for Brockett's model [Brockett, 1993].

Example 1.3 has been first published in [Branicky, 1998]. 


\section{References}

V. Acary and B. Brogliato. Numerical Methods for Nonsmooth Dynamical Systems. Applications in Mechanics and Electronics, volume 35 of Lecture Notes in Applied and Computational Mechanics. Springer Verlag, Berlin Heidelberg, 2008.

A. Alessandri and P. Coletta. Design of Luenberger observers for a class of hybrid linear systems. In Hybrid Systems: Computation and Control, pages 7-18, 2001

R. Alur and D.L. Dill. A theory of timed automata. Theoretical Computer Science, 126:183-235, 1994.

R. Alur and T.A. Henzinger. Real-time logics: complexity and expressiveness. Information and Computation, 104:35-77, 1993.

R. Alur, C. Courcoubetis, N. Halbwachs, T. A. Henzinger, P.-H. Ho, X. Nicollin, A. Olivero, J. Sifakis, and S. Yovine. The algorithmic analysis of hybrid systems. Theoretical Computer Science, 138(1):3-34, 1995.

R. Alur, T. Henzinger, G. Lafferriere, and G.J. Pappas. Discrete abstractions of hybrid systems. Proceedings of the IEEE, 88(2):971-984, 2000.

M. Babaali and M. Egerstedt. Observability for switched linear systems. In Hybrid Systems: Computation and Control, volume 2623 of Lecture Notes in Computer Science. Springer, 2004.

A. Balluchi, L. Benvenuti, M. D. Di Benedetto, and A. L. SangiovanniVincentelli. Design of observers for hybrid systems. In C.J. Tomlin and J.R. Greenstreet, editors, Hybrid Systems: Computation and Control, volume 2289 of Lecture Notes in Computer Science, pages 76-89. SpringerVerlag, Stanford, CA, 2002.

G.I. Bara, J. Daafouz, F. Kratz, and J. Ragot. Parameter dependent state observer design for affine LPV systems. International Journal of Control, 74(16):1601-1611, 2001.

A. Bemporad and M. Morari. Control of systems integrating logic, dynamics, and constraints. Automatica, 35(3):407-427, March 1999. 
A. Bemporad, F. Borrelli, and M. Morari. Piecewise linear optimal controllers for hybrid systems. In Proceedings of the 2000 American Control Conference, pages 1190-1194, Chicago, Illinois, June 2000a.

A. Bemporad, G. Ferrari-Trecate, and M. Morari. Observability and controllability of piecewise affine and hybrid systems. IEEE Transactions on Automatic Control, 45(10):1864-1876, 2000b.

A. Bemporad, A. Garulli, S. Paoletti, and A. Vicino. A bounded-error approach to piecewise affine system identification. IEEE Transactions on Automatic Control, 50(10):1567-1580, 2005.

J. Bengtsson and W. Yi. Timed automata: Semantics, algorithms and tools. In J. Desel, W. Reisig, and G. Rozenberg, editors, Lectures on Concurrency and Petri Nets, volume 3098 of Lecture Notes in Computer Science, pages 87-124. Springer, Berlin, 2004. ISBN 978-3-540-22261-3.

V.D. Blondel and J.N. Tsitsiklis. Complexity of stability and controllability of elementary hybrid systems. Automatica, 35(3):479-490, 1999.

F. Borrelli. Constrained Optimal Control of Linear and Hybrid Systems, volume 290 of Lecture Notes in Control and Information Sciences. Springer, Berlin, 2003. ISBN 3-540-00257-X.

M. S. Branicky, V. S. Borkar, and S. K. Mitter. A unified framework for hybrid control: Model and optimal control theory. IEEE Transactions on Automatic Control, 43(1):31-45, 1998.

M.S. Branicky. Multiple Lyapunov theory and other analysis tools for switched and hybrid systems. IEEE Transactions on Automatic Control, 43(4):475482, 1998.

R.W. Brockett. Hybrid models for motion control systems. In H.L. Trentelman and J.C. Willems, editors, Essays on Control: Perspectives in the Theory and its Applications, volume 14 of Progress in Systems and Control Theory, pages 29-53, Boston, 1993. Birkhäuser.

B. Brogliato, A. Daniilidis, C. Lemaréchal, and V. Acary. On the equivalence between complementarity systems, projected systems and differential inclusions. Systems \& Control Letters, 55:45-51, 2006.

C. Cai, A.R. Teel, and R. Goebel. Smooth lyapunov functions for hybrid systems part i: Existence is equivalent to robustness. IEEE Transactions on Automatic Control, 52(7):1264 - 1277, 2007.

M.K. Camlibel, W.P.M.H. Heemels, and J.M. Schumacher. Consistency of a time-stepping method for a class of piecewise linear networks. IEEE Transactions on Circuits and Systems - I, 49(3):349-357, 2002.

M.K. Camlibel, J.S. Pang, and J. Shen. Conewise linear systems: non-zenoness and observability. SIAM Journal on Control and Optimization, 45:1769$1800,2006$.

M.K. Camlibel, W.P.M.H. Heemels, and J.M. Schumacher. Algebraic necessary and sufficient conditions for the controllability of conewise linear systems. IEEE Transactions on Automatic Control, 53(3):762-774, 2008. 
C.G. Cassandras. Discrete Event Systems: Modeling and Performance Analysis. The Aksen Associates Series in Electrical and Computer Engineering. Richard D. Irwin, Inc., Burr Ridge, Illinois, 1993.

C.G. Cassandras, D.L. Pepyne, and Y. Wardi. Optimal control of a class of hybrid systems. IEEE Transactions on Automatic Control, 46(3):398-415, 2001. ISSN 0018-9286.

Z. Chaochen, C.A.R. Hoare, and A.P. Ravn. A calculus of durations. Information Processing Letters, 40(5):269-276, 1991.

M. Chen, C. R. Zhu, and G. Feng. Linear-matrix-inequality-based approach to $H_{\infty}$ controller synthesis of uncertain continuous-time piecewise linear systems. IEE Proc.-Control Theory Appl., 151(3):295-300, May 2004.

Y. Cho, C.G. Cassandras, and D.L. Pepyne. Forward decomposition algorithms for optimal control of a class of hybrid systems. Int. J. Robust Nonlin. Control, 2:369-394, 2001.

F.H. Clarke, Y.S. Ledyaev, and R.J. Stern. Asymptotic stability and smooth Lyapunov functions. J. Diff. Eq., 149(1):69-114, 1998.

P. Collins and J.H. van Schuppen. Observability of piecewise-affine hybrid systems. In R. Alur and G.J. Pappas, editors, Hybrid Systems: Computation and Control, volume 2993 of Lecture Notes in Computer Science, pages 265279. Springer Verlag, Berlin, 2004.

C.R. Cutler and B.L. Ramaker. Dynamic matrix control - a computer control algorithm. In Proceedings of the 86th AIChE National Meeting, Houston, Texas, April 1979.

R. David and H. Alla. Petri nets for modelling of dynamic systems - a survey. Automatica, 30(2):175-202, 1994.

J. Davies. Specification and proof in real-time CSP. Cambridge University Press, 1993.

B. De Schutter. Optimal control of a class of linear hybrid systems with saturation. SIAM J. Control Optim., 39(3):835-851 (electronic), 2000. ISSN 0363-0129.

B. De Schutter and T.J.J. van den Boom. MPC for continuous piecewise-affine systems. Systems \& Control Letters, 52(3-4):179-192, July 2004.

G. Ferrari-Trecate, D. Mignone, and M. Morari. Moving horizon estimation for hybrid systems. IEEE Transactions on Automatic Control, 47:1663-1676, 2002.

G. Ferrari-Trecate, M. Muselli, D. Liberati, and M. Morari. A clustering technique for the identification of piecewise affine systems. Automatica, 39 (2):205-217, 2003.

A.F. Filippov. Differential Equations with Discontinuous Righthand Sides. Mathematics and Its Applications. Kluwer, Dordrecht, The Netherlands, 1988.

M.R. Garey and D.S. Johnson. Computers and Intractability: A Guide to the Theory of NP-Completeness. W.H. Freeman and Company, San Francisco, 1979. 
R. Goebel and A. Teel. Solutions to hybrid inclusions via set and graphical convergence with stability theory applications. Automatica, 42(4):573-587, April 2006.

L.C.G.J.M. Habets and J.H. van Schuppen. A controllability result for piecewise-linear hybrid systems. In Proceedings of the 2001 European Control Conference, Porto, Portugal, 2001.

W.P.M.H. Heemels and S. Weiland. Input-to-state stability and interconnections of discontinuous dynamical systems. Automatica, 2008.

W.P.M.H. Heemels, J.M. Schumacher, and S. Weiland. Projected dynamical systems in a complementarity formalism. Operations Research Letters, 27 (2):83-91, 2000a.

W.P.M.H. Heemels, J.M. Schumacher, and S. Weiland. Linear complementarity systems. SIAM J. Appl. Math., 60(4):1234-1269, 2000b.

W.P.M.H. Heemels, B. De Schutter, and A. Bemporad. Equivalence of hybrid dynamical models. Automatica, 37(7):1085-1091, July 2001.

T. Henzinger, P. Kopke, A. Puri, and P. Varaiya. What's decidable about hybrid automata. In Proceedings of the 27th Annual Symposium on the Theory of Computing, STOC'95, pages 373-382. ACM Press, 1995.

T.A. Henzinger, P.W. Kopke, A. Puria, and P. Varaiya. What's decidable about hybrid automata? Journal of Computer and System Sciences, 57(1): 94-124, August 1998.

J.P. Hespanha and A.S. Morse. Stabilization of nonholonomic integrators via logic-based switching. Automatica, 35(3):385-393, 1999.

J.P. Hespanha, D. Liberzon, and A.S. Morse. Towards the supervisory control of uncertain nonholonomic systems. In Proc 18th American Control Conf., San Diego, pages 3520-3524, 1999.

C.A.R. Hoare. Communicating sequential processes. Prentice-Hall, 1985.

L.E. Holloway, B.H. Krogh, and A. Giua. A survey of Petri net methods for controlled discrete event systems. Discrete Event Dynamic Systems: Theory and Applications, 7(2):151-190, April 1997.

J. Hu, J. Lygeros, and S.S. Sastry. Towards a theory of stochastic hybrid systems. In Nancy Lynch and Bruce H. Krogh, editors, Hybrid Systems: Computation and Control, number 1790 in LNCS, pages 160-173. SpringerVerlag, Berlin, 2000.

M. Johansson. Piecewise Linear Control Systems. Springer-Verlag, 2003.

M. Johansson and A. Rantzer. Computation of piecewise quadratic Lyapunov functions for hybrid systems. IEEE Transactions on Automatic Control, 43 (4):555-559, April 1998.

A. Lj. Juloski, W.P.M.H. Heemels, G. Ferrari-Trecate, R. Vidal, S. Paoletti, and J.H.G. Niessen. Comparison of four procedures for the identification of hybrid systems. In M. Morari and L. Thiele, editors, Hybrid Systems: Computation and Control, volume 3414 of Lecture Notes in Computer Science, pages 354-369. Springer Verlag, 2005a. 
A. Lj. Juloski, S. Wieland, and W.P.M.H. Heemels. A Bayesian approach to identification of hybrid systems. IEEE Transactions on Automatic Control, 50(10):1520-1533, 2005b.

A.Lj. Juloski, W.P.M.H. Heemels, and S. Weiland. Observer design for a class of piecewise linear systems. International Journal of Robust and Nonlinear Control, 17(15):1387-1404, 2007.

C.M. Kellett and A.R. Teel. Smooth lyapunov functions and robustness of stability for difference inclusions. Systems and Control Letters, 52:395-405, 2004.

G. Lafferriere, G.J. Pappas, and S. Sastry. O-minimal hybrid systems. Math. Control Signals Syst., 13(1):1-21, March 2000.

M. Lazar, W.P.M.H. Heemels, S. Weiland, and A. Bemporad. Stabilizing model predictive control of hybrid systems. IEEE Transactions on Automatic Control, 51(11):1813-1818, 2006.

Y.S. Ledyaev and E.D. Sontag. A Lyapunov characterization of robust stabilization. Nonlinear Analysis, 37:813-840, 1999.

K.K. Lee and A. Arapostathis. On the controllability of piece-wise linear hypersurface systems. Systems and Control Letters, 9(89-96), 1987.

D. Liberzon. Switching in Systems and Control. Systems \& Control: Foundations and Applications. Birkhäuser, Boston, Massachusetts, 2003. ISBN 0-8176-4297-8.

D. Liberzon and A.S. Morse. Basic problems in stability and design of switched systems. IEEE Control Systems Magazine, 19:59-70, 1999.

N. Lynch, R. Segala, F. Vaandrager, and H.B. Weinberg. Hybrid I/O automata. In Hybrid Systems III, volume 1066 of Lecture Notes in Computer Science, pages 496-510. Springer Verlag, 1996.

D.Q. Mayne and S. Raković. Model predictive control of constrained PWA discrete-time systems. International Journal of Robust and Nonlinear Control, 13(3-4):261-279, March-April 2003.

D. Mignone, G. Ferrari-Trecate, and M. Morari. Stability and stabilization of piecewise affine and hybrid systems: an lmi approach. In Proceedings of the 39th IEEE Conference on Decision and Control, Sydney, Australia, 2000.

P. Morin and C. Samson. Robust stabilization of driftless systems with hybrid open-loop/feedback control. In Proc. 19th American Control Conference, Chicago, 2000.

I. Necoara, B. De Schutter, T. van den Boom, and H. Hellendoorn. Min-max model predictive control for uncertain max-min-plus-scaling systems. In Proceedings of the 8th International Workshop on Discrete Event Systems (WODES'06), pages 439-444, Ann Arbor, Michigan, July 2006. ISBN 14244-0053-8.

A. Pavlov, N. van de Wouw, and H. Nijmeijer. Convergent piecewise affine systems: analysis and design. In Proc. CDC/ECC, Sevilla, Spain, 2005.

D.L. Pepyne and C.G. Cassandras. Modeling, analysis, and optimal control of a class of hybrid systems. Discrete Event Dynamic Systems: Theory and Applications, pages 175-201, 1998. 
S. Petterson. Switched state jump observers for switched systems. In Proceedings of the IFAC World Congress, Prague, Czech Republic, 2005.

A. Pnueli. The temporal logic of programs. In Proceedings of the 18th Annual Symposium on the Foundations of Computer Science, pages 46-57. IEEE Computer Science Press, New York, 1977.

G. Pola, M.L. Bujorianu, J. Lygeros, and M.D. Di Benedetto. Stochastic hybrid models: An overview with applications to air traffic management. In Proceedings of the 2003 IFAC Conference on Analysis and Design of Hybrid Systems (ADHS'03, pages 45-50, June 2003.

C. Prieur. Asymptotic controllability and robust asymptotic stabilizability. SIAM J. Control Opt., 43:1888-1912, 2005.

C. Prieur and A. Astolfi. Robust stabilization of chained systems via hybrid control. IEEE Transactions on Automatic Control, 48(10):1768-1772, 2003.

C. Prieur and E. Trélat. Quasi-optimal robust stabilization of control systems. SIAM J. Control Opt., 45(5):1875-1897, 2006.

C. Prieur, R. Goebel, and A.R. Teel. Hybrid feedback control and robust stabilization of nonlinear systems. IEEE Transactions on Automatic Control, 52(11):2103-2117, 2007.

A. Puri, P. Varaiya, and V. Borkar. $\epsilon$-approximation of differential inclusions. In Proceedings of the 34th IEEE Conference on Decision and Control, pages 2892-2897, New Orleans, Louisiana, December 1995.

A. Rantzer and M. Johansson. Piecewise linear quadratic optimal control. IEEE Transactions on Automatic Control, 45(4):629-637, April 2000.

J. Richalet, A. Rault, J.L. Testud, and J. Papon. Model predictive heuristic control: Applications to industrial processes. Automatica, 14(5):413-428, September 1978.

J. Roll, A. Bemporad, and L. Ljung. Identification of piecewise affine systems via mixed-integer programming. Automatica, 40(1):37-50, 2004.

E.D. Sontag. Nonlinear regulation: the piecewise linear approach. IEEE Transactions on Automatic Control, 26(2):346-357, 1981.

E.D. Sontag. Interconnected automata and linear systems: a theoretical framework in discrete-time. In R. Alur, T.A. Henzinger, and E.D. Sontag, editors, Hybrid Systems III, volume 1066 of Lecture Notes in Computer Science, pages 436-448. Springer, 1996.

E.D. Sontag. Mathematical Control Theory: Deterministic Finite Dimensional Systems. Springer, New York, 1998. Texts in applied Mathematics, vol. 6.

G. Stremersch and R.K. Boel. On the influencing net and forbidden state control of timed Petri nets with forced transitions. In Proceedings of the 37th IEEE Conference on Decision and Control, pages 3287-3292, Tampa, Florida, December 1998.

L. Tavernini. Differential automata and their discrete simulators. Nonlinear Analysis, Theory, Methods \& Applications, 11(6):665-683, 1987.

A.J. van der Schaft and J.M. Schumacher. The complementary-slackness class of hybrid systems. Mathematics of Control, Signals and Systems, 9:266-301, 1996. 
A.J. van der Schaft and J.M. Schumacher. Complementarity modelling of hybrid systems. IEEE Transactions on Automatic Control, 43(4):483-490, 1998.

R. Vidal, S. Soatto, and S. Sastry. An algebraic geometric approach for identification of linear hybrid systems. In Proceedings of 42nd IEEE Conference on Decision and Control, 2003.

M. Wicks and R. DeCarlo. Solution of coupled Lyapunov equations for the stabilization of multimodal linear systems. In Proceedings of the American Control Conference, pages 1709-1713, 1997.

H. Ye, A.N. Michel, and L. Hou. Stability theory for hybrid dynamical systems. IEEE Transactions on Automatic Control, 43(4):461-474, April 1998. 